\title{
Aortic root systolic excursion and its association with exercise tolerance in patients with heart failure with preserved ejection fraction
}

\begin{abstract}
Objective: We aimed to investigate the association between aortic root systolic excursion (ARSE) as a simple echocardiographic parameter and exercise tolerance in patients with heart failure with preserved ejection fraction (HFpEF).

Methods: Eighty patients (mean age $57.9 \pm 10.5$ years) with $\mathrm{HFpEF}$ were compared with 80 with age and sex matched healthy subjects. Transthorathic echocardiography was performed with specific assessment of aortic root systolic excursion. Left ventricular longitudinal (LVGLS) and circumferential strain (LVGCS) were evaluated with speckle tracking imaging. In addition all participates underwent 6minute walking test (6MWT).

Results: ARSE was reduced in subject with HFpEF compared with controls $(p<0.05)$. Furthermore, HFpEF patients with $6 \mathrm{MWTD}<300 \mathrm{~m}$ had pronounced decrease in ARSE compared with those with $6 \mathrm{MWTD} \geq 300 \mathrm{~m}$ and control subjects ( $<<0.001)$. 6MWTD was correlated with ARSE $(p<0.001)$, LVGLS $(p<0.001)$ and LVGCS $(p<0.01)$. ARSE had significant correlation with LVGLS $(\mathrm{p}<0.001)$ and LVGCS $(\mathrm{p}<0.003)$. Moreover, ARSE correlated negatively with LAVI $(r=-0.438, p<0.001)$, E/e' ratio $(r=-0.349, p<0.01)$. After multivariate analysis ARSE remained a strong independent predictor of exercise tolerance in patients with $\mathrm{HFpEF}(\mathrm{p}<0.001)$. ROC analysis revealed that ARSE $\leq 7.5 \mathrm{~mm}$ was the optimal cut-off value to predict reduced exercise intolerance in HFpEF patients $(\mathrm{AUC}=0.91 ; \mathrm{p}<0.001)$

Conclusion: We found that, patients with HFpEF have reduced ARSE, which was significantly associated reduced 6MWTD. Reduced ARSE was correlated with subclinical LV systolic dysfunction and diastolic dysfunction. We suggest that ARSE, as a simple echocardiographic parameter might be of value, in order to better discriminate HFpEF patients risk profile.
\end{abstract}

Keywords: aortic root, heart failure, subclinical LV dysfunction; STE, speckle tracking echocardiography, LVGCS, left ventricular global circumferential strain
Volume 14 Issue 6 - 202 I

\author{
Ragab A Mahfouz, Marwa M Gad, Mohamed \\ Arab and Mohei Abulfoutoh \\ Cardiology Department, Zagazig University Hospital, Egypt
}

Correspondence: Ragab A Mahfouz, Professor of Cardiology, Zagazig University Hospital, Egypt, Tel, 0020 I00642767I, Email ragabaziza6।@yahoo.com, ragabaziza6I@gmail.com

Received: October 05, 202I | Published: November 23, 202 |
Abbreviations: HFpEF, heart failure with preserved ejection fraction; ARSE, aortic root systolic excursion

\section{Introduction}

Heart failure with preserved ejection fraction (HFpEF) is a major public health problem, accounting for $50 \%$ of HF admissions, ${ }^{1}$ with comparable mortality and morbidity to heart failure with reduced ejection fraction. ${ }^{2} \mathrm{HFpEF}$ is a heterogeneous disease, with multiple probable mechanisms underlying the clinical syndrome. ${ }^{3}$

Definitely, HFpEF still considered a medical challenge, as there is no complete understanding of its pathogenesis. Furthermore, in HFpEF subjects, several reports identified various echocardiographic predictors of medical importation, ${ }^{4,5}$ quality of lifetime, ${ }^{6}$ and exercise tolerance.

Anatomically, the root of aorta is a conduit between the LV and ascending aorta. Anterior and posterior walls of aorta move anterior during systolic phase and posterior during diastolic phase. $^{8}$

Several studies investigated the association between aortic root systolic excursion and left ventricular systolic functional indices. These studies reported that ARSE may represent an echocardiographic marker for left ventricular systolic, especially when Global strain is not achievable or apical views are not available. Furthermore, aortic root has high echogenicy and ARSE can easily be imagined by M-mode echocardiography., 90

However, the ARSE in patients with HFpEF is not clearly investigated. We hypothesized that aortic root systolic excursion (ARSE) as a simple echocardiographic marker, could predict exercise tolerance in subjects with HFpEF. Hence, our aim was to explore the relation of ARSE with exercise tolerance and its association with subclinical systolic dysfunction in HFpEF patients.

\section{Subjects \& methods}

Eighty subjects with HFpEF were enrolled for the research (mean age $53.5 \pm 11.3 \mathrm{y}, 45 \%$ of them were female). The study included patients with: (1) typical symptoms of HF; (2) LVEF more than 50\%; (3) abnormal LV relaxation, blunted early mitral annular velocity (e'); high E/e' and B-type natriuretic peptide (BNP) $>400 \mathrm{pg} / \mathrm{ml} .^{11,12}$ They were compared with 80 healthy subjects, who matched with patients in age and sex.

We excluded patients with Acute Myocardial Infarction, unstable angina, pacemaker implantation, dilated left ventricle, cardiomyopathy, and valvular heart problems, atrial fibrillation, chronic obstructive and 
possible lung disease regardless the type of pulmonary hypertension, liver disorders, chronic kidney disease, thyroid diseases, arthritis, and patients unable to underwent 6 minute walking test. All participates gave an informed written consent and the study was approved by the faculty scientific and ethical committee.

\section{Resting two-dimensional echocardiography}

Transthoracic echocardiographic assessment was performed for all subjects with the use of Vivid 9, General Electric Healthcare (GE Vingmed, Norway) equipped with a harmonic M5S variablefrequency $(2.5-4 \mathrm{MHz})$ phased-array transducer. All participants were evaluated by the same operator. The obtained parameters included left ventricular ejection fraction (LVEF \%), and the left ventricular mass index. Normal left ventricular mass index was considered, when it was $95 \mathrm{~g} / \mathrm{m}^{2}$ in women and $115 \mathrm{~g} / \mathrm{m}^{2}$ in men. Left atrium diameter was indexed for body surface area to calculate LA volume index (LAVI $\mathrm{ml} / \mathrm{m}^{2}$ ). Echocardiographic systolic pulmonary artery pressure (SPAP) was calculated as previously described. ${ }^{13,14}$

Aortic root systolic excursion (ARSE) was assessed by placing M-mode cursor on center of the root of aorta (Figure 1). The amplitude of systolic movement of anterior aortic wall was calculated to represent ARSE (mm). The average of 3 measures were obtained from the far wall in the long parasternal axis view..$^{15}$

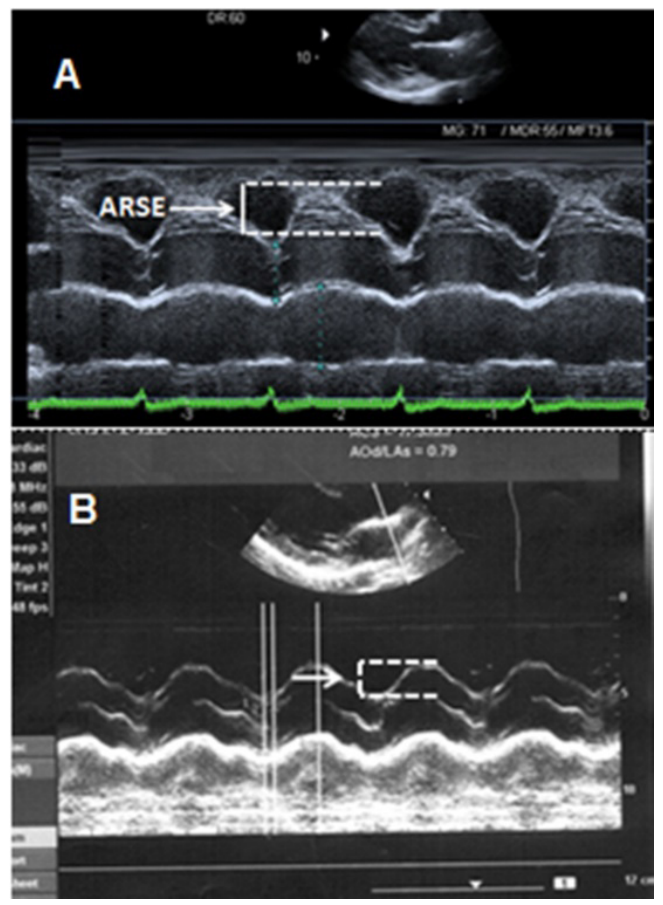

Figure I A- Aortic root systolic excursion (ARSE) in patients with good exercise tolerance $(6 M W T D \geq 300 \mathrm{~m})$. B- ARSE in patients with reduced exercise tolerance $(6 \mathrm{MWTD}<300 \mathrm{~m})$.

\section{Speckle tracking echocardiography (STE)}

Two- dimensional gray scale imaging was achieved. Apical twoand four-chamber views were used to assess left ventricular global longitudinal strain (LVGLS). Then we turned to parasternal shortaxis views at left ventricular base and mid-level to evaluate left ventricular global circumferential strain (LVGCS). The endocardial border was traced manually at end systole. The entire left ventricular circumference was divided into 6 segments and generated myocardial strain curves by frame-by-frame tracking of the natural acoustic markers throughout the cardiac cycle. With frame rate of 60 to $80 \mathrm{frames} / \mathrm{s}$. and at the end of expiratory breath hold, images were acquired and transferred to a workstation for further offline analysis. The offline analysis was performed using automated software On a GE® EchoPAC workstation. Only myocardial segment with good quality by the operator and automatic system were used for analysis. ${ }^{16}$

\section{Six-minute walking test "6-MWT"}

According to Guyatt et al. ${ }^{17}$ protocol, participates undertook 6MWT. We explained everything with respect to the test for all participates and they were informed to take their drugs. Subjects inquired to walk without any support as far as they can in fifteen obstacle-free hallway, rotating $180^{\circ}$ at end of the hallway throughout allotted six minutes. Participates terminated the test, if they experienced any worrying symptoms or signs. Total walking distance was measured and symbolized in meters.

\section{Statistical analyses}

Measurement values are expressed as mean \pm SD for continuous variables and were compared with two-tailed Student $t$ test. While, qualitative data were expressed as number and percentage and were analyzed by Chi-square (X2) test. Pearson coefficients analysis was used for correlations between variables. We underwent univariate analysis to recognize predictors of six minute walking test distance. Whilst, we underwent multivariate logistic regression to discriminate independent predictors for 6MWTD. All statistical studies were achieved with the use of commercially available SPSS (Version-21).

\section{Results}

Table 1 depicts the demographic characteristics of HFpEF subjects versus healthy subjects. All basic data were similar, except for brain natriuretic peptide $(\mathrm{BNP})$, which was higher $(\mathrm{P}<0.01)$ in patients with HFpEF. In addition, six-minute walking test distance (6MWTD) was significantly decreased in HFpEF patients $(\mathrm{p}<0.001)$.

With respect to echocardiographic data, Table 2 revealed that, patients with $\mathrm{HFpEF}$ had abnormal LV diastolic function [lower e' $(\mathrm{P}<0.001)$; increased E/e' ratio $(\mathrm{P}<0.001)$ and increased LAVI $(\mathrm{P}<0.001)]$ compared with controls. Furthermore, ARSE considerably decreased $(\mathrm{P}<0.001)$ in HFpEF subjects than healthy subjects. As far as STE findings, the results showed that both LVGLS and LGCS decreased in the HFpEF patients ( $\mathrm{P}<0.01$ and $<0.03$ singly).

Table I Characteristics of patients with heart failure with preserved ejection fraction versus controls

\begin{tabular}{llll}
\hline Variable & HFpEF patients $\mathbf{n}=\mathbf{8 0}$ & Control group $\mathbf{n}=\mathbf{8 0}$ & P value \\
\hline Age, $y$ & $57.9 \pm 10.5$ & $55.3 \pm 9.7$ & 0.10 \\
Men, $\mathrm{n}(\%)$ & $59(73.8 \%)$ & $60(75)$ & 0.57 \\
Body mass index $(\mathrm{kg} / \mathrm{m} 2)$ & $27.5 \pm 3.1$ & $25.8 \pm 3.0$ & 0.15 \\
Smokers, $\mathrm{n}(\%)$ & $32(40)$ & $35(43.8)$ & $0.5 \mathrm{I}$ \\
Hypertension, $\mathrm{n}(\%)$ & $62(77.5)$ & -- & \\
\hline
\end{tabular}




\begin{tabular}{|c|c|c|c|}
\hline Variable & HFpEF patients $n=80$ & Control group $n=80$ & $P$ value \\
\hline Diabetes, n(\%) & $43(53.8)$ & -- & \\
\hline Coronary artery disease(\%) & $19(23.8 \%)$ & -- & \\
\hline Heart rate(beats/min) & $75 \pm 7$ & $70 \pm 8$ & 0.09 \\
\hline Systolic BP(mm Hg) & $135 \pm 22$ & $130 \pm 20$ & 0.13 \\
\hline Diastolic BP(mm Hg) & $82 \pm 11$ & $78 \pm 12$ & 0.35 \\
\hline Blood glucose(mg/dL) & $128 \pm 11$ & $107 \pm 5$ & $<0.05$ \\
\hline Total cholesterol(mg/dL) & $208 \pm 31$ & $191 \pm 29$ & 0.28 \\
\hline LDL-C(mg/dL) & $135 \pm 23$ & $128 \pm 25$ & 0.14 \\
\hline $\mathrm{HDL}-\mathrm{C}(\mathrm{mg} / \mathrm{dL})$ & $43 \pm 8$ & $4 \mid \pm 7$ & 0.62 \\
\hline Triglycerides(mg/dL) & $158 \pm 39$ & $|39 \pm 4|$ & 0.11 \\
\hline Creatinine & $1.2 \pm 0.4$ & $0.95 \pm 0.21$ & 0.22 \\
\hline Brain natriuretic peptide $(\mathrm{pg} / \mathrm{mL})$ & $208.6 \pm 41.5$ & $35.8 \pm 12.15$ & $<.0 .01$ \\
\hline 6 MWT distance, $m$ & $395 \pm 32$ & $638 \pm 41$ & $<0.001$ \\
\hline
\end{tabular}

LDL-C, low-density lipoprotein-cholesterol; HDL-C, high-density lipoprotein-cholesterol; 6 MWTD, Six minute walking test distance

Table 2 Echocardiographic parameters in HFpEF patients versus control subjects

\begin{tabular}{llll}
\hline & HFpEF patients $\mathbf{n = 8 0}$ & Control group $\mathbf{n}=\mathbf{8 0}$ & P value \\
\hline LA volume index $(\mathrm{mL} / \mathrm{m} 2)$ & $35.9 \pm 4.8$ & $21.5 \pm 3.7$ & $<0.003$ \\
LV mass index(g/m2) & $96.6 \pm 17.5$ & $77.3 \pm 15.2$ & 0.13 \\
LV EF\%(\%) & $61.9 \pm 3.5$ & $64.5 \pm 5.3$ & 0.29 \\
E wave & $81.3 \pm 4.7$ & $61.3 \pm 2.3$ & 0.17 \\
S' wave $^{\prime}$ & $6.82 \pm 1.30$ & $6.91 \pm 1.35$ & 0.25 \\
e' wave & $7.23 \pm 0.21$ & $11.58 \pm 0.25$ & $<0.01$ \\
E/e' ratio & $12.5 \pm 4.3$ & $5.1 \pm 1.7$ & $<0.001$ \\
SPAP, mmHg & $43 \pm 14$ & $21 \pm 6$ & $<0.01$ \\
ARSE, mm & $9.52 \pm 1.80$ & $12.65 \pm 2.21$ & 0.01 \\
LVGLS(\%) & $-17.65 \pm 2.95$ & $-21.15 \pm 3.74$ & $<0.01$ \\
LVGLSR-s $(s-1)$ & $-1.31 \pm 0.19$ & $-1.53 \pm 0.19$ & $<0.05$ \\
LVGCS(\%) & $-16.09 \pm 4.10$ & $-19.32 \pm 3.45$ & $<0.03$ \\
LVGCSR-s(s-l) & $-1.35 \pm 0.18$ & $-1.62 \pm 0.21$ & $<0.05$ \\
\hline
\end{tabular}

LAVI, Left atrial volue index; LV, left ventricle; LVEF, left ventricular ejection fraction; LVGLS, Left ventricular global longitudinal strain; LVGLSR, Left ventricular global longitudinal strain rate; LVGCSR, Left ventricular global circumferential strain rate; LVGCSR, Left ventricular global circumferential strain rate

We categorized patients with HFpEF into two groups according to the results of 6MWT distance; group with reduced exercise tolerance, included $36(45 \%)$ subjects with six MWT distance $<300 \mathrm{~m}$ and group with good exercise tolerance, included 44(55\%) subject with six MWT distance $\geq 300 \mathrm{~m}$. Table 3 represents the baseline data of HFpEF groups. Patients with reduced 6MWTD had increased heart rate $(\mathrm{P}<0.05)$, higher systolic blood pressure $(\mathrm{P}<0.03)$, high $\mathrm{BNP}(\mathrm{P}<0.03)$. Furthermore, reduced exercise tolerance group has 6MWTD $256 \pm 19$, versus $531 \pm 41$ of group with good exercise tolerance.

Table 3 Characteristics of patients with HFpEF with 6 MWTD $<300 \mathrm{~m}$ versus those with 6 MWTD $\geq 300 \mathrm{~m}$. preserved ejection fraction versus controls

\begin{tabular}{llll}
\hline Variable & HFpEF with 6MWTD<300 $\mathbf{~} \mathbf{~ n = 3 6}$ & HFpEF with 6MWTD $\geq \mathbf{3 0 0} \mathbf{~ m ~} \mathbf{n}=\mathbf{4 4}$ & P value \\
\hline Age, $y$ & $59.3 \pm 11.5$ & $56.8 \pm 10.5$ & 0.09 \\
Men, $\mathrm{n}(\%)$ & $28(77.8 \%)$ & $3 \mathrm{I}(70.5)$ & 0.25 \\
Body mass index $(\mathrm{kg} / \mathrm{m} 2)$ & $27.3 \pm 3.0$ & $26.9 \pm 3.2$ & 0.27 \\
Smokers, $\mathrm{n}(\%)$ & $16(44.4)$ & $18(40.9)$ & 0.65 \\
\hline
\end{tabular}




\begin{tabular}{|c|c|c|c|}
\hline Variable & HFpEF with $6 M W T D<300 \mathrm{~m} \mathrm{n}=36$ & HFpEF with $6 M W T D \geq 300 \mathrm{~m} \mathrm{n}=44$ & $P$ value \\
\hline Hypertension, n(\%) & $29(77.5)$ & $33(75)$ & 0.74 \\
\hline Diabetes, $\mathrm{n}(\%)$ & $20(53.8)$ & $23(52.3)$ & 0.81 \\
\hline Coronary artery disease(\%) & $9(25 \%)$ & $10(22.7)$ & 0.57 \\
\hline Heart rate(beats/min) & $88 \pm 13$ & $73 \pm 8$ & $<0.05$ \\
\hline Systolic BP(mm Hg) & $139 \pm 22$ & $127 \pm 19$ & $<0.03$ \\
\hline Diastolic BP(mm Hg) & $82 \pm 11$ & 81 & 0.35 \\
\hline Blood glucose(mg/dL) & $|28 \pm| \mid$ & $125 \pm 13$ & 0.36 \\
\hline Total cholesterol(mg/dL) & $210 \pm 35$ & $202 \pm 41$ & 0.46 \\
\hline LDL-C(mg/dL) & $138 \pm 26$ & $132 \pm 2 \mid$ & 0.37 \\
\hline $\mathrm{HDL}-\mathrm{C}(\mathrm{mg} / \mathrm{dL})$ & $42 \pm 7$ & $45 \pm 6$ & 0.52 \\
\hline Triglycerides(mg/dL) & $163 \pm 38$ & $157 \pm 40$ & 34 \\
\hline Creatinine & $1.19 \pm 0.45$ & $|.08 \pm 0.3|$ & 0.31 \\
\hline Brain natriuretic peptide $(\mathrm{pg} / \mathrm{mL})$ & $295.6 \pm 49.5$ & $104.5 \pm 24.2$ & $<0.03$ \\
\hline Diuretic, $n(\%)$ & $25(69.4)$ & $31(40.5)$ & 0.58 \\
\hline ACE-I, n(\%) & $21(58.3)$ & $25(58.8)$ & 0.98 \\
\hline ARB, $n(\%)$ & $10(27.8)$ & $\mathrm{II}(25)$ & 0.75 \\
\hline Beta-blocker, n(\%) & $15(41.1)$ & $19(43.2)$ & 0.61 \\
\hline $\mathrm{CCB}, \mathrm{n}(\%)$ & $5(13.8)$ & $5(11.4)$ & 0.45 \\
\hline Nitrates, n(\%) & $5(13.8)$ & $7(15.9)$ & 0.52 \\
\hline 6 MWT distance, $\mathrm{m}$ & $256 \pm 19$ & $53| \pm 4|$ & $<0.001$ \\
\hline
\end{tabular}

LDL-C, low-density lipoprotein-cholesterol; HDL-C, high-density lipoprotein-cholesterol; ACE-ls, angiotensin convertase enzyme inhibitors; ARBs, Angiotensin receptor blockers; $\mathrm{CCB}$, calcium channel blockers

Table 4 depicts the echocardiographic characteristics of patients with 6 MWT $<300 \mathrm{~m}$ versus subjects with six MWT distance $\geq 300 \mathrm{~m}$. Patients with reduced exercise tolerance had decreased e' wave $(\mathrm{P}<0.01)$, increased LAVI $(\mathrm{p}<0.01)$, high $\mathrm{E} / \mathrm{e}^{\prime} \quad(\mathrm{P}<0.001)$ versus $\mathrm{HFpEF}$ patients with $6 \mathrm{MWTD} \geq 300 \mathrm{~m}$. As regards the LVGLS and
LVGCS, we found that HFpEF patients with $6 \mathrm{MWTD}<300 \mathrm{~m}$ had lower values than those with $6 \mathrm{MWTD} \geq 300 \mathrm{~m} \quad(\mathrm{P}<0.001)$. Figure 2 showed that HFpEF patients had significant decrease in ARSE compared with patients with $6 \mathrm{MWTD} \geq 300 \mathrm{~m}$ and control subjects.

Table 4 Echocardiographic parameters in HFpEF patients according to the results of 6 MWT

\begin{tabular}{llll}
\hline & HFpEF with 6MWTD $\geq \mathbf{3 0 0} \mathbf{~} \mathbf{n}=\mathbf{4 4}$ & HFpEF with 6MWTD<300 $\mathbf{~} \mathbf{n}=\mathbf{3 6}$ & $\mathbf{P}$ value \\
\hline LA volume index $(\mathrm{mL} / \mathrm{m} 2)$ & $32.5 \pm 3.3$ & $39.8 \pm 5.7$ & $<0.01$ \\
LV mass index $(\mathrm{g} / \mathrm{m} 2)$ & $95.8 \pm 16.5$ & $97.5 \pm 19.5$ & 0.63 \\
LV EF\%(\%) & $62.9 \pm 3.3$ & $61.2 \pm 3.1$ & 0.49 \\
E wave & $71.5 \pm 3.3$ & $89.2 \pm 5.1$ & 0.26 \\
S' wave & $6.85 \pm 1.2$ & $6.79 \pm 1.2$ & 0.22 \\
e' wave & $7.92 \pm 0.20$ & $6.15 \pm 0.11$ & $<0.01$ \\
E/e' ratio & $9.1 \pm 3.5$ & $16.5 \pm 4.7$ & $<0.001$ \\
SPAP; mmHg & $47 \pm 13$ & $41 \pm 11$ & 0.10 \\
ARSE; mm & $11.54 \pm 2.13$ & $7.21 \pm 1.52$ & $<0.001$ \\
LVGLS(\%) & $-19.93 \pm 3.55$ & $-15.83 \pm 2.57$ & $<0.001$ \\
LVGLSR-s(s-l) & $-1.49 \pm 0.17$ & $-1.09 \pm 0.15$ & $<0.03$ \\
LVGCS(\%) & $-18.95 \pm 4.61$ & $-13.01 \pm 3.05$ & $<0.001$ \\
LVGCSR-s(s-l) & $-1.55 \pm 0.22$ & $-1.07 \pm 0.16$ & $<0.01$ \\
\hline
\end{tabular}

LAVI, left atrial volue index; LV, left ventricle; LVEF, left ventricular ejection fraction; LVGLS, left ventricular global longitudinal strain; LVGLSR, Left ventricular global longitudinal strain rate; LVGCSR, Left ventricular global circumferential strain rate; LVGCSR, Left ventricular global circumferential strain rate 


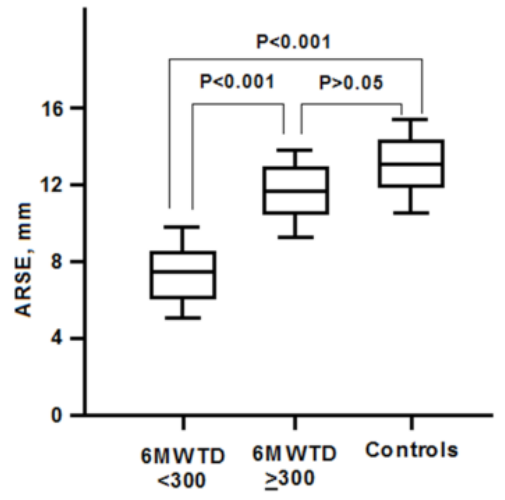

Figure 2 The box graph that shows aortic root systolic excursion in patients with HFpEF and 6MWTD $<300 \mathrm{~m}$ compared with those with 6MWTD $\geq 300 \mathrm{~m}$ and control subjects.

Our results showed a significant correlation of $6 \mathrm{MWTD}$ with ARSE $(\mathrm{P}<0.001)$, LVGLS\% $(\mathrm{P}<0.001)$, and LVGCS\% $(\mathrm{P}<0.001)$. Contrarily, it was inversely associated with $\mathrm{E} / \mathrm{e}$ ' $(\mathrm{P}<0.02)$ (Table 5). Likewise, using ARSE as a dependent factor, the correlation analysis (Table 6) revealed that ARSE had strong relation with LVGLS $(\mathrm{r}=0.59 ; \mathrm{P}<0.001)$, whilst, it modestly correlated with $\mathrm{E} / \mathrm{e}$ ' and LAVI and LVGCS $(\mathrm{P}<0.01)$.

Table 5 Correlation analysis between 6MWD and different echocardiographic parameters in $\mathrm{HFpEF}$ patients

\begin{tabular}{lll}
\hline Variable & $\boldsymbol{r}$ & P value \\
\hline LV mass index (g/m2.7) & -0.13 & 0.19 \\
LV EF(\%) & 0.17 & 0.11 \\
Aortic root systolic excursion & 0.62 & $<0.001$ \\
LAVl; ml/m2 & -0.42 & $<0.01$ \\
e' wave & 0.25 & 0.13 \\
S' wave & 0.27 & 0.11 \\
E/e' & -0.35 & $<0.01$ \\
LVGLS(\%) & 0.61 & $<0.001$ \\
LVGCS(\%) & 0.52 & $<0.01$
\end{tabular}

LVEF, Left ventricular ejection fraction; LAVI, left atrial volume index; LVGLS, Left ventricular global longitudinal strain; LVGCS, Global circumferential strain rate

Table 6 Correlation between ARSE and echocardiographic parameters in HFpEF patients

\begin{tabular}{lll}
\hline Variable & $\boldsymbol{r}$ & P value \\
\hline LV mass index(g/m2.7) & 0.11 & 0.25 \\
LV EF(\%) & 0.21 & 0.06 \\
LAVI; ml/m2 & -0.43 & $<0.02$ \\
e' wave & 0.25 & $<0.05$ \\
S' wave $^{\prime}$ & 0.25 & $>0.05$ \\
Ele' & -0.39 & $<0.02$ \\
LVGLS(\%) & 0.59 & $<0.001$ \\
LVGCS(\%) & 0.46 & $<0.01$
\end{tabular}

LVEF, left ventricular ejection fraction; LAVI, left atrial volume index; LVGLS, left ventricular global longitudinal strain; LVGCS, Global circumferential strain rate
Multivariate analysis revealed that ARSE and LVGLS and LVGCS were observed to be significant independent predictors of decreased exercise capacity in patients with HFpEF (Table 7).

Table 7 Univariate and multivariate echo predictors for reduced exercise capacity in patients with heart failure with preserved ejection fraction

\begin{tabular}{lllll}
\hline & Univariate & \multicolumn{3}{l}{ Multivariate } \\
\hline Variable & OR(95\%C.I) & P value & OR(95\%C.I) & P value \\
LVEF\% & $I .05(0.99-I .08)$ & $0.4 I$ & -- & -- \\
ARSE & $I .73(I . I 3-2.4 I)$ & $<0.00 I$ & $I .92(I . I 3-2.7 I)$ & $<0.00 I$ \\
LAVI; ml/m2 & $I .06(I .0 I-2.98)$ & $<0.03$ & $1.09(0.98-I .75)$ & 0.21 \\
E/e' & $I .38(0.95-1.75)$ & $<0.05$ & $0.83(0.95-1.19)$ & 0.15 \\
LVGLS & $I .83(0.80-2.93)$ & $<0.00 I$ & $1.75(0.78-2.7 I)$ & $<0.00 I$ \\
LVGCS & $I .65(0.83-2.45)$ & $<0.003$ & $1.43(0.8 I-2.15)$ & $<0.01$ \\
\hline
\end{tabular}

LVEF, left ventricular ejection fraction; ARSE, aortic root systolic excursion; LAVI, left atrial volume index; LVGLS, left ventricular global longitudinal strain; LVGCS, global circumferential strain rate

ROC analysis was done to detect the best cut-off point to predict exercise intolerance in subjects with heart failure with preserve ejection fraction. Results showed the ARSE $\leq 7.5 \mathrm{~mm}$ was best cutoff value for predicting exercise intolerance (Figure 3 ). AUC $=0.91$, with sensitivity of $93 \%$, specificity of $96 \%$.

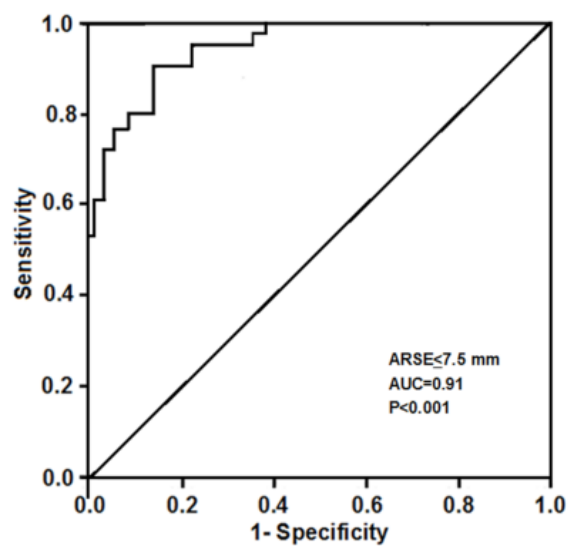

Figure 3 ROC curve analysis for aortic root systolic excursion to predict exercise intolerance $(6 \mathrm{MWTD}<300 \mathrm{~m})$ in HFpEF patients.

\section{Discussion}

The present research revealed: (1) $45 \%$ of patients with $\mathrm{HFpEF}$ had $6 \mathrm{MWTD}<300 \mathrm{~m}$, (2) HFpEF patient with $6 \mathrm{MWTD}<300 \mathrm{~m}$ had lower ARSE as compared with those with 6 MWTD $\geq 300 \mathrm{~m}$, (3) significant correlations between ARSE and LV global longitudinal and circumferential systolic strain, whilst ARSE was inversely associated with LAVI, E/e' \& BNP, (4) ARSE was an independent predictor of reduced exercise tolerance, and (5) importantly, ARSE $\leq 7.5 \mathrm{~mm}$ was the optimal value for predicting reduced exercise capacity in subjects with HFpEF.

Importantly, our findings revealed that LVGLS and LVGCS in HFpEF patients was lower than control subjects and the decrease was pronounced in subjects with exercise intolerance, implies that HFpEF subjects reduced exercise tolerance had significant subclinical systolic dysfunction. This might be related with presence of more progressive disease process with subclinical systolic dysfunction. Also, straight mechanical ventricular-arterial link affords another justification for 
the detected association between ARSE and left ventricular systolic performance.

Assessment of systolic displacement of aorta is an earliest deed of echocardiography and spot the starting imaging of functional evaluation of the heart. Nevertheless, principal mechanisms are not fully understood. Aurich $\mathrm{M}$, et al. ${ }^{18}$ assumed, that aortic systolic excursion might be largely produced by movement of base to the apex of the heart and subsequently could be considered a marker of longitudinal function of left ventricle. Moreover, they supposed that ARSE would be related mainly to left ventricular systolic function. Nevertheless, our findings demonstrated that ARSE also, was associated with E/e' and LAVI. There is no clear underlying pathogenesis, could explain this relationship. However, higher left ventricular filling pressure resulted an increase in LA wall stress and stiffening (structural remodeling of LA) and accordingly, lessens emptying, augment wall stress with decrease compliance and increases BNP secretion. ${ }^{19,20}$

The reduced ARSE in HFpEF patients with reduced exercise tolerance could be explained by the presence of subclinical systolic dysfunction that could be unmasked with exercise, as our results revealed that ARSE was significantly associated with LV global longitudinal and circumferential systolic strain. Moreover, severe diastolic dysfunction may relate to more progressive disease process, as fibrotic changes, endothelial dysfunction and consequently more impaired contraction and systolic dysfunction.

Aortic recoil possibly, may affect the resistance to the empty of left atrium, reduction pressure, and consequently, aid atrial-ventricular Pressure gradient in physiological conditions. Moreover, recoil of aorta during diastole, following systolic stretch, could ease left ventricular filling and ejections. ${ }^{21}$ Nevertheless, stiffening of aorta and decreasing its systolic excursion, the valuable influence of stretching and aortic wall recoil to the initial ventricular filling during diastole might be lessened. ${ }^{22}$

Pratt et al. ${ }^{23}$ investigated the posterior aortic wall motion displacement with cardiac hemodynamics and established strong relation with left ventricular stroke volume, and they concluded that ASRE is a reaction to the entire left ventricular performance. Moreover, the beginning of ARSE matches with pressure increase and current velocity in the ascending aorta and therefore imitates hemodynamic changes due to contraction of left ventricle. ${ }^{24}$ Furthermore, it was suggested that posterior aortic wall displacement is mainly influenced by volume changes of LA. ${ }^{25,26}$

Importantly, the current research showed a negative association between ARSE and both E/e' \& BNP level in HFpEF. A finding that provides the value of ARSE in predicting not only, exercise intolerance but also the severity of diastolic dysfunction as well as sbclinical left ventricular systolic dysfunction in HFpEF. A previous study suggested that variations in left ventricular and aortic physiology provide a significant impact in disposition to HF, including HFpEF. This study revealed a significant correlation between stiff aorta and left ventricular global longitudinal performance. ${ }^{27}$ Furthermore, Kaess et al. assumed that left ventricular diastolic dysfunction as well as more stiffening of aorta might play a significant role left ventricular global longitudinal performance. ${ }^{29}$

\section{Limitation}

First, single center study with small sample number. Second, the aortic root evaluation motion parameters were obtained utilizing two-D imaging of the PLAX plane. Aortic root movement pathway achieved from parasternal short axis view, where the aortic root is clearly visible. Third, we don't use the 3D motion. The 3D motion information can also be obtained by tracking the aortic root directly on volumetric data available in 3D echocardiography. Finally, intraobserver or inter-observer variability testing was not performed. Finally, most patients in our cohort had pulmonary hypertension in comparison to the control; it would be expected that those population would have exercise intolerance. This compromises the external validity of the study.

\section{Conclusions}

We have found that reduced ARSE was significantly associated decreased 6MWTD in patients with HFpEF. These findings concurrently linked to high left ventricular filling pressure, subclinical left ventricular systolic dysfunction and higher values of brain natriuretic peptide. Hence, we suggest that ARSE might be a simple echocardiographic parameter in predicting exercise capacity in cardiac patients, who have normal ejection fraction.

\section{Conflicts of interest}

All authors declared that they have no conflict of interest.

\section{Acknowledgments}

None.

\section{Conflicts of interest}

None.

\section{Funding}

None.

\section{References}

1. Meagher P, Adam M, Civitarese R, et al. Heart failure with preserved ejection fraction in diabetes: mechanisms and management. Can $J$ Cardiol. 2018;34(5):632-643.

2. Dunlay SM, Roger VL, Redfield MM. Epidemiology of heart failure with preserved ejection fraction. Nat Rev Cardiol. 2017;14(10):591-602.

3. Borlaug BA. The pathophysiology of heart failure with preserved ejection fraction. Nat Rev Cardiol. 2014;11(9):507- 515.

4. Donal E, Lund LH, Oger E, et al. New echocardiographic predictors of clinical outcome in patients presenting with heart failure and a preserved left ventricular ejection fraction: a subanalysis of the Ka (Karolinska) Ren (Rennes) Study. Eur J Heart Fail. 2015;17(7):680- 688.

5. Pirozzi F, Paglia A, Sasso L, et al. Mitral peak early diastolic filling velocity to deceleration time ratio as a predictor of prognosis in patients with chronic heart failure and preserved or reduced ejection fraction. $J$ Geriatr Cardiol. 2015;12(4):346-352.

6. Ahmeti A, Henein MY, Ibrahimi P, et al. Quality of life questionnaire predicts poor exercise capacity only in HFpEF and not in HFrEF. BMC Cardiovasc Disord. 2017;17(1):268.

7. Batalli A, Ibrahimi P, Bytyçi I, et al. Different determinants of exercise capacity in HFpEF compared to HFrEF. Cardiovasc Ultrasound. 2017;15:12.

8. Hagendorff A, Stoebe S, Tayal B. A systematic approach to 3D echocardiographic assssment of the aortic root. Glob Cardiol Sci Pract. 2018;(2):12.

9. Aurich M, Niemers M, Fuchs P, et al. Pathophysiological background and prognostic implication of systolic aortic root motion in non-ischemic dilated. Sci Rep. 2019;9(1):3866. 
10. Jingi AM, Hamadou B, Noubiap JJ, et al. Correlations of left ventricular systolic function indices with aortic root systolic excursion (ARSE): A cross-sectional echocardiographic study. PLoS One. 2018;13(11):e0206199

11. Kosmala W, Rojek A, Przewlocka-Kosmala M, et al. Contributions of Nondiastolic Factors to Exercise Intolerance in Heart Failure with Preserved Ejection Fraction. J Am Coll Cardiol. 2016;67(6):659-670.

12. Mcmurray JJ, Adamopoulos S, Anker SD, et al. ESC Guidelines for the diagnosis and treatment of acute and chronic heart failure 2012: The Task Force for the Diagnosis and Treatment of Acute and Chronic Heart Failure 2012 of the European Society of Cardiology. Developed in collaboration with the Heart Failure Association (HFA) of the ESC. Eur Heart $J$ 2012;13(14):1787-1847.

13. Kircher BJ, Himelman RB, Schiller NB. Noninvasive estimation of right atrial pressure from the inspiratory collapse of the inferior vena cava. $\mathrm{Am}$ J Cardiol. 1990;66(4):493-496.

14. Lang RM, Badano LP, Mor-Avi V, et al. Recommendations for cardiac chamber quantification by echocardiography in adults: An update from the american society of echocardiography and the European association of cardiovascular imaging. J Am Soc Echocardiogr. 2015;28(1):1-39.e14.

15. Aurich M, Niemers M, Fuchs P, et al. Pathophysiological background and prognostic implication of systolic aortic root motion in non-ischemic dilated cardiomyopathy. Sci Rep. 2019;9(1):3866.

16. Geyer H, Caracciolo G, Abe H, et al. Assessment of myocardial mechanics using speckle tracking echocardiography: fundamentals and clinical applications. J Am Soc Echocardiogr. 2010;23(4):351-369.

17. Guyatt GH, Sullivan MJ, Thompson PJ, et al. The 6-minute walk: a new measure of exercise capacity in patients with chronic heart failure. Can Med Assoc J. 1985;132(8):919-923.

18. Sherif F Nagueh SF, Smiseth OA, Appleton CP, et al. Recommendations for the Evaluation of Left Ventricular Diastolic Function by Echocardiography: An Update from the American Society of Echocardiography and the European Association of Cardiovascular Imaging. J Am Soc Echocardiogr. 2016;29(4):277-314.
19. Aurich M, Niemers M, Fuchs P, et al. Pathophysiological background and prognostic implication of systolic aortic root motion in non-ischemic dilated cardiomyopathy. Sci Rep. 2019;9(1):3866.

20. O’Connor K, Magne J, Rosca M, et al. Left atrial function and remodelling in aortic stenosis. Eur J Echocardiogr. 2011;12(4):299-305.

21. Miyoshi H, Oishi Y, Mizuguchi Y, et al. Effect of an increase in left ventricular pressure overload on left atrial-left ventricular coupling in patients with hypertension: a two-dimensional speckle tracking echocardiographic study. Echocardiography. 2013;30(6):658-666.

22. 21. Bell V, Sigurdsson 1S, Westenberg JJM, et al. Relations between aortic stiffness and left ventricular structure and function in older participants in the Age, Gene/Environment Susceptibility-Reykjavik Study. Circ Cardiovasc Imaging. 2015;8(4):e003039.

23. Bell V, Mitchell GF. Influence of vascular function and pulsatile hemodynamics on cardiac function. Curr Hypertens Rep. 2015;17(9):580.

24. Pratt RC, Parisi AF, Harrington JJ, et al. The Influence of Left Ventricular Stroke Volume on Aortic Root Motion: An Echocardiographic Study. Circulation. 1976;53(6):947-953.

25. Chandraratna PAN, Chu W, Schechter E, et al. Hemodynamic Correlates of Echocardiographic Aortic Root Motion* Observations on Normal Subjects and Patients with Idiopathic Hypertrophic Subaortic Stenosis. Chest. 1978;74:183-189.

26. Strunk BL, Fitzgerald JW, Lipton M, et al. The Posterior Aortic Wall Echocardiogram, Its Relationship to Left Atrial Volume Change. Cardiovasc Res. 1976;54(5):744-750.

27. Biamino G, Wessel HJ, Schlag W, et al. Echocardiographic pattern of motion of the aortic root as a correlate of left atrial volume changes. $\mathrm{Am}$ Heart J. 1980;100(2):191-200.

28. Bell V, McCabe EL, Larson MG, et al. Relations between aortic stiffness and left ventricular mechanical function in the community. J Am Heart Assoc. 2017;6(1):e004903.

29. Kaess BM, Rong J, Larson MG, et al. Relations of central hemodynamics and aortic stiffness with left ventricular structure and function: the Framingham Heart Study. J Am Heart Assoc. 2016;5(3):e002693. 